\title{
Ginsenoside Rg1 protects against neuronal degeneration induced by chronic dexamethasone treatment by inhibiting NLRP-1 inflammasomes in mice
}

\author{
YAODONG ZHANG $^{1 *}$, WEN HU $^{1 *}$, BIQIONG ZHANG ${ }^{1 *}$, YANYAN YIN $^{1}$, \\ JUNYAN ZHANG ${ }^{1}$, DAKE HUANG ${ }^{2}$, RONGRONG HUANG ${ }^{3}$, WEIPING LI ${ }^{1}$ and WEIZU LI ${ }^{1}$ \\ ${ }^{1}$ Department of Pharmacology, Key Laboratory of Anti-Inflammatory and Immunopharmacology, \\ Ministry of Education; ${ }^{2}$ Synthetic Laboratory of Basic Medicine College, Anhui Medical University, \\ Hefei, Anhui 230032; ${ }^{3}$ Department of Pharmacology, Anhui Xinhua University, Hefei, Anhui 230088, P.R. China
}

Received February 11, 2017; Accepted July 28, 2017

DOI: $10.3892 / \mathrm{ijmm} .2017 .3092$

\begin{abstract}
Glucocorticoids (GCs) are known to alter neuronal plasticity, impair learning and memory and play important roles in the generation and progression of Alzheimer's disease. There are no effective drug options for preventing neuronal injury induced by chronic GC exposure. Ginsenoside Rg1 $(\operatorname{Rg} 1)$ is a steroidal saponin found in ginseng. The present study investigated the neuroprotective effect of Rg1 on neuroinflammation damage induced by chronic dexamethasone $(5 \mathrm{mg} / \mathrm{kg}$ for 28 days) exposure in male mice. Our results showed that $\mathrm{Rg} 1$ ( 2 and $4 \mathrm{mg} / \mathrm{kg}$ ) treatment increased spontaneous motor activity and exploratory behavior in an open field test, and increased the number of entries into the new object zone in a novel object recognition test. Moreover, $\mathrm{Rg} 1$ (2 and $4 \mathrm{mg}$ / $\mathrm{kg}$ ) treatment significantly alleviated neuronal degeneration and increased MAP2 expression in the frontal cortex and hippocampus. Additionally, inhibition of NLRP-1 inflammasomes was also involved in the mechanisms underlying the effect of Rg1 on GC-induced neuronal injury. We found that $\mathrm{Rg} 1$ ( 2 and $4 \mathrm{mg} / \mathrm{kg}$ ) treatment increased the expression of glucocorticosteroid receptor and decreased the expression of NLRP-1, ASC, caspase-1, caspase-5, IL-1 $\beta$ and IL-18 in the hippocampus in male mice. The present study indicates that Rg1 may have protective effects on neuroinflammation and neuronal injury induced by chronic GC exposure.
\end{abstract}

Correspondence to: Dr Weizu Li, Department of Pharmacology, Key Laboratory of Anti-Inflammatory and Immunopharmacology, Ministry of Education, Anhui Medical University, 81 Meishan Road, Hefei, Anhui 230032, P.R. China

E-mail: liweizu@126.com

${ }^{*}$ Contributed equally

Key words: glucocorticoids, ginsenoside Rg1, locomotor behavior, NLRP1 inflammasome, neurodegeneration

\section{Introduction}

Alzheimer's disease (AD) is a progressive and neurodegenerative disorder with dementia resulting from the combination of both genetic and environmental risk factors (1). Several studies have linked psychosocial stress and stress hormone levels, such as glucocorticoids (GCs), with AD generation and development $(2,3)$. Clinical studies have shown that plasma cortisol levels are significantly increased in AD and mild cognitive impairment patients, and dysfunction of the hypothalamus-pituitary-adrenal (HPA) axis is associated with a higher risk of AD (4). Furthermore, it has been reported that the rate of dementia progression is correlated with the plasma levels of GCs in AD patients (3). GCs are known to alter neuronal plasticity, impair learning and memory, produce atrophy in several areas of the brain, reduce hippocampal dendritic complexity $(5,6)$, and promote hippocampal cell death (7). Yet, the precise mechanisms by which they contribute to AD remain to be fully elucidated (8).

Currently, there are no effective drugs for preventing neuronal damage induced by chronic GC exposure. Ginseng has been safely used to improve health conditions, and delay senescence for more than 2,000 years in China. Ginsenosides are the major active components in ginseng. It has been reported that ginsenosides have neuroprotective effects in animals and humans $(9,10)$. Ginsenoside $\operatorname{Rg} 1$ (Rg1) is a steroidal saponin which is abundantly found in ginseng. Recent studies have revealed that $\operatorname{Rg} 1(2.5-10 \mathrm{mg} / \mathrm{kg})$ prevents spatial learning and memory impairment, and may be a useful agent for neurodegenerative disorders such as AD (11). However, the protective effect of $\operatorname{Rg} 1$ on neuronal inflammation damage induced by chronic GC exposure and the underlying mechanisms have not yet been fully elucidated. Moreover, mifepristone (RU486) is a synthetic glucocorticosteroid receptor (GR) antagonist (12). RU486 has been used as a tool to study the mechanisms of steroid receptor function (13). Recent studies have reported that RU486 may act as a neuroprotective agent against excitotoxicity and traumatic brain injury $(14,15)$. In the present study, RU486 was used as a positive control. 
Dexamethasone (DEX) is a type of synthetic glucocorticoid. DEX $(5 \mathrm{mg} / \mathrm{kg})$ is often used to mimic GC-induced neurodegenerative diseases $(16,17)$. Our most recent study showed that chronic DEX $(5 \mathrm{mg} / \mathrm{kg})$ exposure for 28 days significantly induced neurodegeneration and increased neuroinflammation via NLRP-1 inflammasome activation (18). A previous study by us also demonstrated that $\operatorname{Rg} 1$ protects against learning and memory impairments and ROS oxidative damage in chronic restraint stress-exposed mice (19). ROS oxidative stress is a method by which to activate NLRP-1 inflammasomes. In the present study we hypothesized that Rg1 inhibits the activation of NLRP-1 inflammasomes and attenuates neuronal damage induced by chronic DEX exposure. Our results indicated that $\mathrm{Rg} 1$ has protective effects against neuronal inflammation damage induced by chronic DEX (5 mg/kg, 28 days) exposure.

\section{Materials and methods}

Animals and treatment. All experiments were carried out according to protocols approved by the Commitee on the Care and Use of Laboratory Animals at Anhui Medical University. Adult male ICR mice (25-30 g, Grade II) were obtained from the Center for Laboratory Animals of Anhui. The mice were housed in groups of four to six with unrestricted access to food and water and a 12-h light/dark cycle.

Animals were randomly divided into six groups (10 mice in each group): control group, DEX (5 mg/kg) exposure group, DEX $(5 \mathrm{mg} / \mathrm{kg})+$ RU486 $(5 \mathrm{mg} / \mathrm{kg})$ treatment group and DEX $(5 \mathrm{mg} / \mathrm{kg})+\mathrm{Rg} 1(1,2,4 \mathrm{mg} / \mathrm{kg})$ treatment groups. DEX (Sigma, St. Louis, MO, USA) was dissolved in alcohol at a concentration of $500 \mathrm{mg} / \mathrm{ml}$ to be used as a stock solution and diluted in normal saline (NS) at a concentration of $0.5 \mathrm{mg} / \mathrm{ml}$ before use. RU486 (Beijing Zizhu Pharmaceutical Co., Ltd., Beijing, China) was prepared by dissolving RU486 in distilled water at a concentration of $0.5 \mathrm{mg} / \mathrm{ml}$. The $\operatorname{Rg} 1$ (content of $\operatorname{Rg} 1>98 \%$, obtained from the Institute of Pharmaceutical Research of Xiehe Medical University of China) was dissolved in distilled water at concentrations of $0.1,0.2$ and $0.4 \mathrm{mg} / \mathrm{ml}$. In the DEX, RU486 and Rg1 treatment groups, the mice were injected subcutaneously (s.c.) with DEX (5 mg/kg/day) for 28 days. In the control group, the mice were injected (s.c.) with NS with an equal volume of alcohol. The RU486 $(5 \mathrm{mg} / \mathrm{kg})$ group was treated with RU486 solution $(0.5 \mathrm{mg} / \mathrm{ml})$ intragastrically (i.g., $0.1 \mathrm{ml} / 10 \mathrm{~g})$ for 28 days. The $\operatorname{Rg} 1(1,2,4 \mathrm{mg} / \mathrm{kg})$ groups were treated with $\operatorname{Rg} 1(0.1,0.2$ and $0.4 \mathrm{mg} / \mathrm{ml})$ intragastrically (i.g., $0.1 \mathrm{ml} / 10 \mathrm{~g}$ ) for 28 days. The control group and DEX exposure received equivalent volumes of distilled water for 28 days.

Open field test. The open field test was performed to study the effects of Rg1 on DEX exposure-induced motor activity and exploratory behavior impairments. It is often used to detect the locomotor behavior, anxiety and exploration of animals (20). The open field test apparatus (ShangHai Biowill Co., Ltd., Shanghai, China) consisted of a computer-tracking cage $(60 \times 60 \times 50 \mathrm{~cm})$ which was separated as 9 squares $(1$ central and 8 peripheral) by two vertical lines and transverse lines as previously described (21). The mouse was placed in the cage for $2 \mathrm{~min}$ in each testing period. The ANY-maze Behavioral Tracking Software (Stoelting Co., Wood Dale, IL, USA) was used to record the path of its motor activity and exploration for
$3 \mathrm{~min}$. The number of line crossings, the moving distance $(\mathrm{m})$, the mean moving speed $(\mathrm{m} / \mathrm{sec})$ and the number of standing up events (indicates exploratory behavior) were calculated by the software to represent the motor and exploration behaviors (22).

Novel object recognition (NOR) test. The NOR test is usually used to evaluate recognition memory (23). The open field apparatus and the ANY-maze Behavioral Tracking system are used to detect the NOR task. The NOR test consists of three phases: the habituation phase, the familiarization phase, and the test phase. In the habituation phase, the mice are placed in an empty arena to adapt to the environment. The next day, the mice are placed in the familiar arena with two identical objects (a1, a2) (Fig. 2A). Twenty-four hours after familiarization, the mice are placed in the open field with (a1) the familiar object and (b) a novel object to examine long-term recognition memory. The number of entries to $\mathrm{a} 2$ zone and the time exploring the $\mathrm{a} 2$ zone in the familiarization phase, the number of entries to $\mathrm{b}$ zone, and the time exploring the $\mathrm{b}$ zone in the test phase were recorded to evaluate the effects of $\operatorname{Rg} 1$ on long-term recognition memory impairments.

Histological examination. The mice were anesthetized by injection of $3.5 \%$ chloral hydrate (i.p., $0.1 \mathrm{ml} / 10 \mathrm{~g}$ body weight). The brains $(n=5)$ were carefully removed and fixed in $4 \%$ paraformaldehyde. The brain tissues were embedded in paraffin and sliced into $5-\mu \mathrm{m}$ sections using a section cutter (Leica, Mannheim, Germany). The sections were stained with hematoxylin and eosin (H\&E) and the neuronal morphology in the frontal cortex and hippocampus CA1 and CA3 was observed by a microscope (Olympus IX71; Olympus, Tokyo, Japan).

Immunohistochemistry. The immunohistochemical analysis was performed as previously described (18). The brain sections were deparaffinized and incubated with $3 \%$ hydrogen peroxide and then blocked with non-immune goat serum. The sections were washed with PBS and incubated in the mouse anti-MAP2 antibody (1:200; ab11268; Abcam, Cambridge, UK) overnight at $4^{\circ} \mathrm{C}$. The next day, the sections were washed 3 times with PBS, and incubated in the secondary antibody for $1 \mathrm{~h}$. Then the sections were washed and visualized by using an $\mathrm{ABC}$ kit (Beijing Zhongshan Golden Bridge Biotechnology Co., Ltd., Beijing, China). The sections were mounted and examined by a light microscope (Olympus IX71; Olympus). The positive neurons were stained brown. Image-Pro Plus 6.0 analysis system was used to analyze the mean optical density in the frontal cortex, hippocampus CA1 and CA 3 by an observer blinded to the experimental protocol.

Immunoblot analysis. The immunoblot method was performed according to our previous description (18). The total protein in the frontal cortex and hippocampus was extracted. Equal amounts $(40 \mu \mathrm{g})$ of protein were transferred to polyvinylidene difluoride (PVDF) membranes. The membranes were blocked with 5\% skim milk in TBS-T for $1 \mathrm{~h}$ at room temperature. The membranes were washed with TBS-T 3 times, and incubated with primary antibodies for NLRP-1, ASC, caspase-1, caspase-5, IL-1 $\beta$, IL-18, glucocorticoid receptor (GR) and $\beta$-actin $(1: 1,000)$ overnight at $4^{\circ} \mathrm{C}$. The antibodies for ASC 

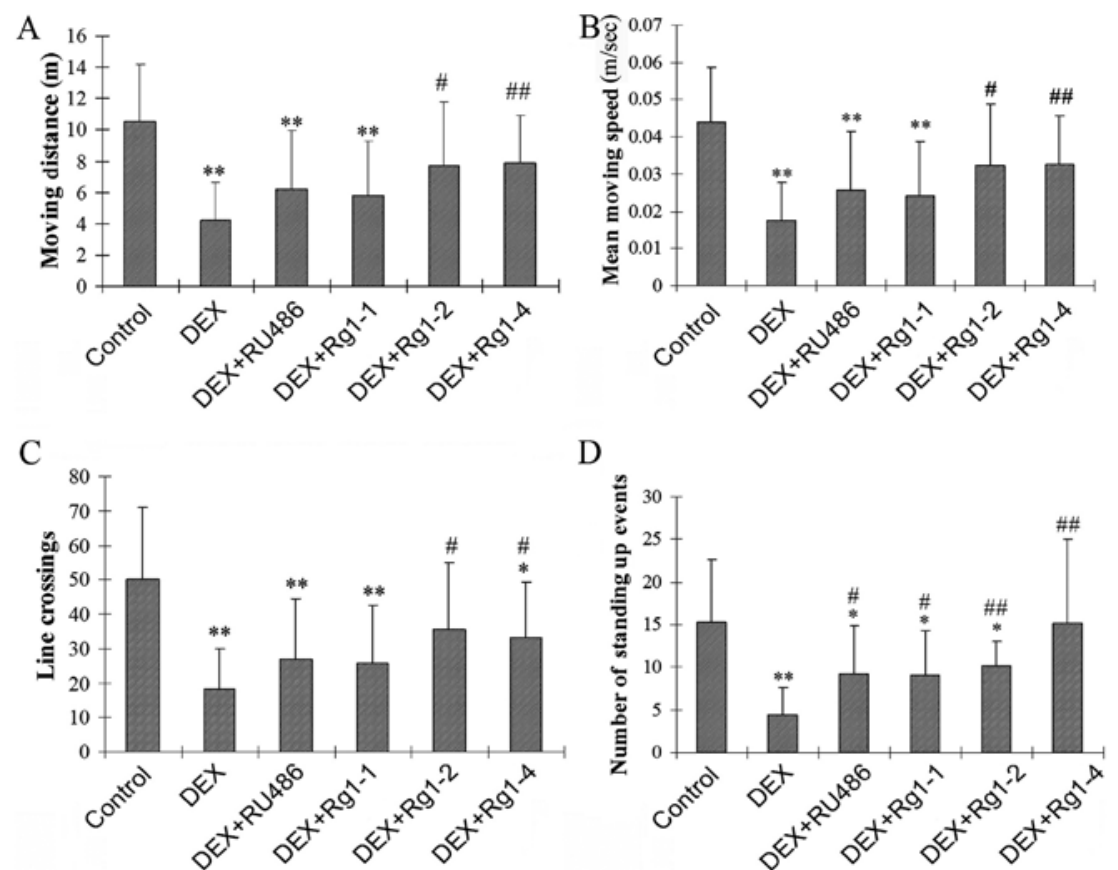

Figure 1. Effects of ginsenoside Rg1 (Rg1) on motor activity and exploratory behavior in chronic DEX-exposed mice (open field test). (A) The total moving distance, (B) the mean moving speed, (C) the number of line crossings and (D) the number of standing up events. $\mathrm{Rg} 1(2 \mathrm{and} 4 \mathrm{mg} / \mathrm{kg})$ treatment increased the total moving distance, the mean moving speed, the number of line crossings and the number of standing up events in male mice. Results are expressed as mean $\pm \mathrm{SD}, \mathrm{n}=10,{ }^{*} \mathrm{P}<0.05,{ }^{* *} \mathrm{P}<0.01$ compared withthe control group; ${ }^{\#} \mathrm{P}<0.05,{ }^{\# \#} \mathrm{P}<0.01$ compared with the DEX-exposed group. DEX, dexamethasone.

(BS2215), caspase-1 (BS1730), caspase-5 (BS7048), IL-1 (BS3506) and GR (BS6617) were provided by Bioworld Technology, Inc. (St. Louis Park, MN, USA). The NLRP-1 antibody (ab16091) was provided by Abcam. The IL-18 antibody (sc-6179) was obtained from Santa Cruz Biotechnology, Inc. (Dallas, TX, USA). The dilution of all primary antibodies was at 1:500. On the next day, the membranes were washed 3 times with TBS-T and incubated with HRP-conjugated secondary antibody $(1: 5,000)$ for $1 \mathrm{~h}$. After the membranes were extensively washed, the proteins were detected using the ECL kit (Amersham Biosciences, Little Chalfont, UK). The protein bands were visualized using Chemi Q4800 Mini Imaging System (Bioshine, Shanghai, China), and Image J Software [National Institutes of Health (NIH), Bethesda, MD, USA] was used for densitometry and quantification of the protein bands. The immunoreactive band intensities were normalized to its corresponding band of $\beta$-actin.

Statistical analysis. All data are reported as mean \pm SD. Statistical differences were performed using SPSS 17.0. The data were analyzed by one-way ANOVA followed by Bonferroni's post hoc test for between-group comparisons. $\mathrm{P}<0.05$ was considered to indicate a statistically significant difference.

\section{Results}

Effects of Rg1 on motor and exploratory behavior impairments in mice induced by chronic DEX exposure. In the present study, the open field test was used to examine motor and exploratory behavior. The results showed that DEX exposure significantly decreased the motor activity and exploratory behavior in male mice. DEX exposure for 28 days significantly decreased the total moving distance $(\mathrm{m})$, the mean moving speed $(\mathrm{m} / \mathrm{sec})$, the number of line crossings, and the number of standing up movements (Fig. 1A-D, P<0.01). Rg1 (2 and $4 \mathrm{mg} / \mathrm{kg}$ ) treatment significantly increased the total $\mathrm{m}$, the mean $\mathrm{m} / \mathrm{sec}$, the number of line crossings, and the number of standing up events in the male mice which were decreased by chronic DEX exposure (Fig. 1A-D, $\mathrm{P}<0.05$ or $\mathrm{P}<0.01$ ).

Effects of Rgl on chronic DEX-induced NOR in mice. The NOR test results showed that, in the familiarization phase, DEX exposure for 28 days had no significant effects on the number of entries into the a 2 zone and the exploring time in the a 2 zone, which is the novel object zone (b zone) in the test phase. While in the test phase, DEX exposure significantly reduced the number of entries into the new object zone (b zone) and the exploring time in the b zone. Compared with the DEX exposure group, RU486 and Rg1 ( 2 and $4 \mathrm{mg} / \mathrm{kg}$ ) treatment increased the number of entries into the $b$ zone and the exploring time in the $\mathrm{b}$ zone in the final test phase (Fig. $2 \mathrm{~B}$ and $\mathrm{C}, \mathrm{P}<0.05$ or $\mathrm{P}<0.01$ ).

Effects of Rgl on chronic DEX-induced neuronal damage in the hippocampus and frontal cortex. The H\&E staining showed that no significant neuronal abnormalities were observed in the frontal cortex and hippocampus CA1 and CA3 in the control group (Fig. 3A). While in the DEX treatment group (Fig. 3B), the neurons showed significant nuclear condensation and acidophilia degeneration. Compared with the DEX treatment group, RU486 and Rg1 ( 2 and $4 \mathrm{mg} / \mathrm{kg}$ ) treatment significantly alleviated the neuronal damage (Fig. 3C, E and F).

Effects of Rg1 on MAP2 expression in the frontal cortex and hippocampus in mice. The results showed that MAP2 expres- 


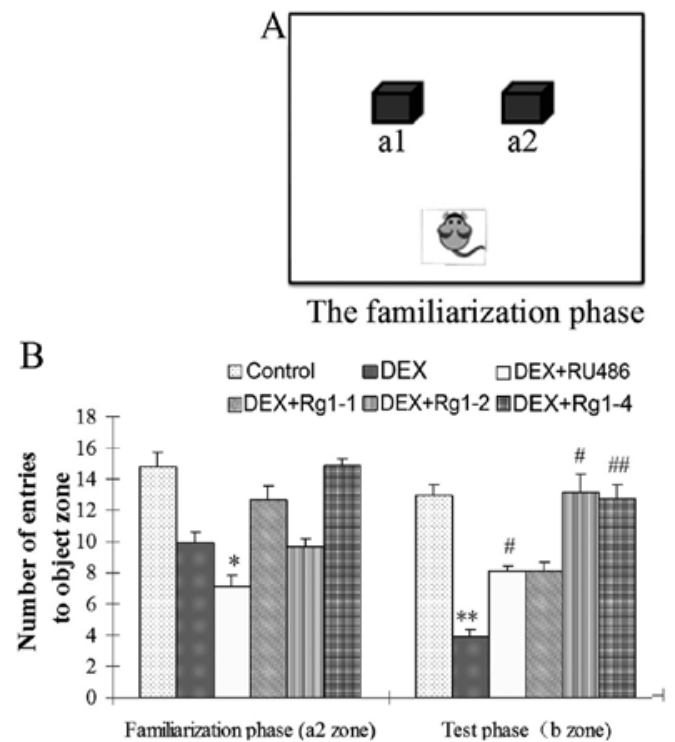

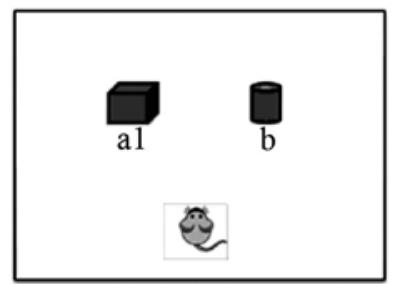

The test phase

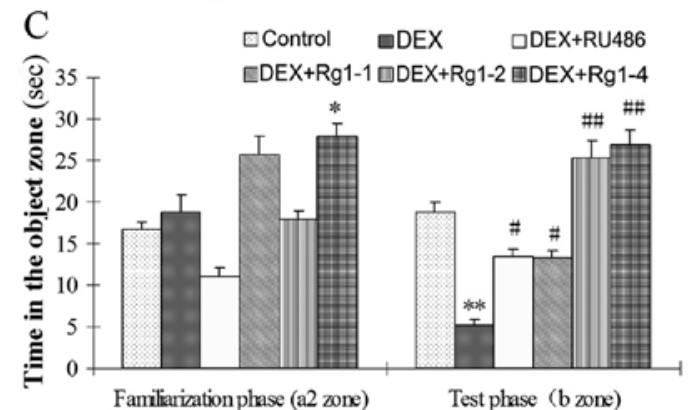

Figure 2. Effects of ginsenoside Rg1 (Rg1) on novel object recognition in chronic DEX-exposed mice (NOR test). (A) The diagram of the NOR test. (B) The number of entries to the object zone. (C) The exploring time in the object zone. In the familiarization phase, DEX exposure for 28 days had no significant effects on the number of entries into the $\mathrm{a} 2$ zone and the time in the $\mathrm{a} 2$ zone. While in the test phase, DEX exposrue significantly reduced the number of entries into the new object zone (b zone) and the time in the b zone. Compared with the DEX exposure group, RU486 and Rg1 ( 2 and $4 \mathrm{mg} / \mathrm{kg})$ treatment increased the number of entries into the $b$ zone and the time in the $b$ zone. Results are expressed as the mean $\pm \mathrm{SD}, \mathrm{n}=10,{ }^{*} \mathrm{P}<0.05,{ }^{* *} \mathrm{P}<0.01$ compared with the control group; $\mathrm{P}<0.05$, ${ }^{\# \#} \mathrm{P}<0.01$ compared with the DEX exposure group. DEX, dexamethasone; NOR, novel object recognition.

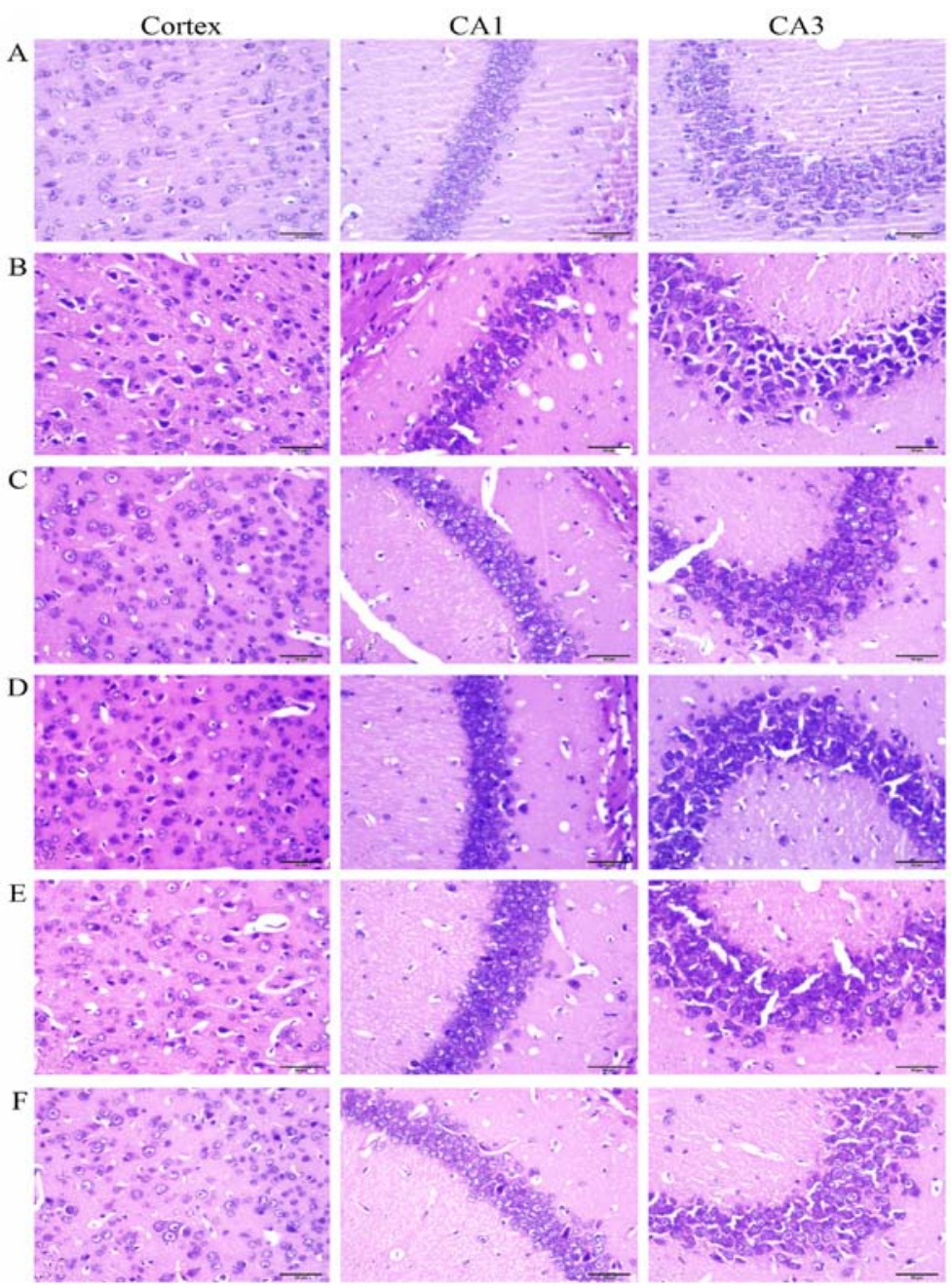

Figure 3. Effects of ginsenoside $\operatorname{Rg} 1(\operatorname{Rg} 1)$ on neuronal injury in the frontal cortex and hippocampus in chronic DEX-exposed mice (H\&E staining, $\mathrm{x} 400)$. Compared with the (A) control group, (B) DEX treatment for 28 days accelerated neuronal injury. Compared with the DEX treatment group, RU486 and Rg1 ( 2 and $4 \mathrm{mg} / \mathrm{kg}$ ) treatment alleviated the neuronal degeneration (C, E and F). (A) Control group; (B) DEX group; (C) DEX+RU486 group; (D) DEX+Rg1 (1 mg/ $\mathrm{kg}$ ) group; (E) DEX+Rg1 (2 mg/kg) group; (F) DEX+Rg1 (4 mg/kg) group. DEX, dexamethasone. 




Figure 4. Effects of ginsenoside Rg1 (Rg1) on MAP2 expression in frontal cortex and hippocampus induced by chronic DEX exposure (immunohistochemistry). (A) Control group; (B) DEX group; (C) DEX+RU486 group; (D) DEX+Rg1 (1 mg/kg) group; (E) DEX+Rg1 (2 mg/kg) group; (F) DEX+Rg1 (4 mg/kg) group; (G) quantitative analysis of MAP2 expression. The results showed that MAP2 protein was abundantly expressed in the frontal cortex and hippocampus in the control group (A). Chronic DEX exposure significantly decreased MAP2 expression in the frontal cortex and hippocampus (B). Compared with the DEX exposure group, treatment with RU486 and Rg1 ( 2 and $4 \mathrm{mg} / \mathrm{kg}$ ) significantly increased MAP2 expression in the frontal cortex and hippocampus. Results are expressed as mean $\pm \mathrm{SD}, \mathrm{n}=6$. ${ }^{* *} \mathrm{P}<0.01$ compared with the control group; ${ }^{\#} \mathrm{P}<0.05,{ }^{\# \#} \mathrm{P}<0.01$ compared with the DEX exposure group. DEX, dexamethasone.

sion was abundant in the frontal cortex and hippocampus in the control group (Fig. 4A and G). In the DEX treatment group, the MAP2 expression was significantly decreased in the frontal cortex and hippocampus (Fig. 4B and G, P<0.01). Compared with the DEX treatment group, RU486 and Rg1 (2 and $4 \mathrm{mg} / \mathrm{kg}$ ) significantly increased the expression of MAP2 in the frontal cortex and hippocampus CA1 and CA3 (Fig. 4C and $\mathrm{E}-\mathrm{G}, \mathrm{P}<0.05$ or $\mathrm{P}<0.01$ ).

Effects of Rgl on GR expression in the frontal cortex and hippocampus. We further detected GR expression in the frontal cortex and hippocampus brain tissue by immunoblot analysis. The results revealed that GR expression was abundant in the hippocampus and cortex in the control groups. In the DEX treatment group, GR expression was significantly decreased (Fig. 5, P<0.01). Compared with the DEX treatment group, RU486 and $\operatorname{Rg} 1$ ( 2 and $4 \mathrm{mg} / \mathrm{kg}$ ) treatment significantly increased the expression of GR (Fig. 5, P $<0.05$ or $\mathrm{P}<0.01$ ).

Effects of Rgl on expression levels of NLRP-1, ASC, caspase-1, caspase-5, IL-1 $\beta$ and $I L-18$ in the frontal cortex and hippocampus. To confirm whether $\mathrm{Rg} 1$ can regulate 
A

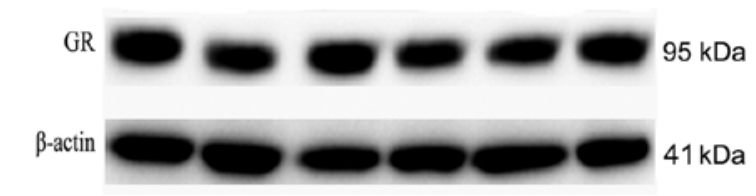

B

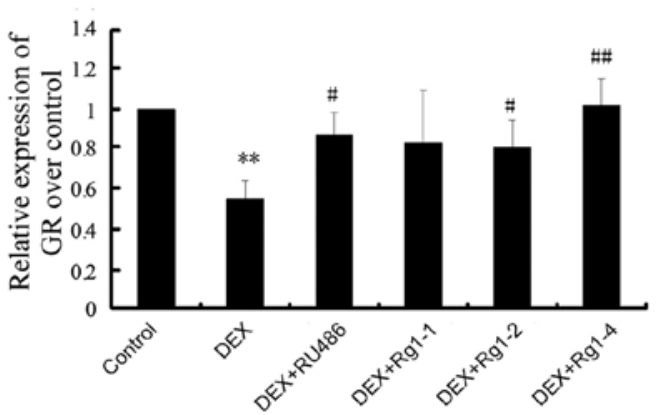

Figure 5. Effects of ginsenoside Rg1 (Rg1) on GR expression in the frontal cortex and hippocampus in chronic DEX-exposed mice (immunoblotting). (A) The immunoblot result of GR and (B) quantitative analysis of GR expression over the control. Results are expressed as mean $\pm \mathrm{SD}, \mathrm{n}=3 .{ }^{* *} \mathrm{P}<0.01$ compared with the control group. ${ }^{\#} \mathrm{P}<0.05,{ }^{\# \#} \mathrm{P}<0.01$ compared with the DEX exposure group. GR, glucocorticosteroid receptor; DEX, dexamethasone.

NLRP1 inflammasome activation, which is involved in chronic DEX-induced hippocampal neuron damage, we further detected the expression of NLRP-1, ASC, caspase-1, caspase-5, IL-1 $\beta$ and IL-18 in the frontal cortex and hippocampus brain tissue by immunoblotting. The results showed that DEX exposure for 28 days significantly increased the expression of NLRP-1 and ASC (Fig. 6, $\mathrm{P}<0.05$ and $\mathrm{P}<0.01$ ), caspase-1 and caspase-5 (Fig. 7, $\mathrm{P}<0.05$ and $\mathrm{P}<0.01$ ) and IL-1 $\beta$ and IL-18 (Fig. 8, $\mathrm{P}<0.01$ ). Compared with the DEX treatment group, RU486 and Rg1 (2 and $4 \mathrm{mg} / \mathrm{kg}$ ) treatment decreased the expression of NLRP-1 and ASC (Fig. 6, P $<0.05$ ), caspase-1 and caspase-5 (Fig. 7, $\mathrm{P}<0.05$ and $\mathrm{P}<0.01$ ) and $\mathrm{IL}-1 \beta$ and IL-18 (Fig. $8, \mathrm{P}<0.05$ or $\mathrm{P}<0.01$ ). These data indicate that $\mathrm{Rg} 1$ decreased the activation of NLRP1 inflammasomes activated by chronic DEX exposure.

\section{Discussion}

A growing number of reports suggest that neuronal inflammation is a contributing factor to neurodegenerative diseases, such as $\mathrm{AD}$ (24-26). It has been reported that inflammasomes play an important role in many neurodegenerative diseases such as Parkinson's disease (PD) and AD (27). The present study was designed to investigate the effects of the ginseng extract ginsenoside $\mathrm{Rg} 1$ on neuroinflammation damage in the frontal cortex and hippocampus in mice following chronic DEX exposure. In the present study, the results showed that $\operatorname{Rg} 1$ ( 2 and $4 \mathrm{mg} / \mathrm{kg}$ ) and RU486 (GR antagonist) treatment increased spontaneous motor activity and exploratory behavior in an open field test, and increased the number of entries into the new object zone in the NOR test. $\mathrm{Rg} 1$ (2 and $4 \mathrm{mg} / \mathrm{kg}$ ) and RU486 significantly protected against neuronal damage induced by chronic DEX exposure. Rg1 ( 2 and $4 \mathrm{mg} / \mathrm{kg}$ ) and RU486 were able to decrease the expression of NLRP-1 inflammasomes and attenuate the neuronal damage in the frontal cortex and hippocampus in mice. Additional, Rg1 and RU486 treatment increased GR
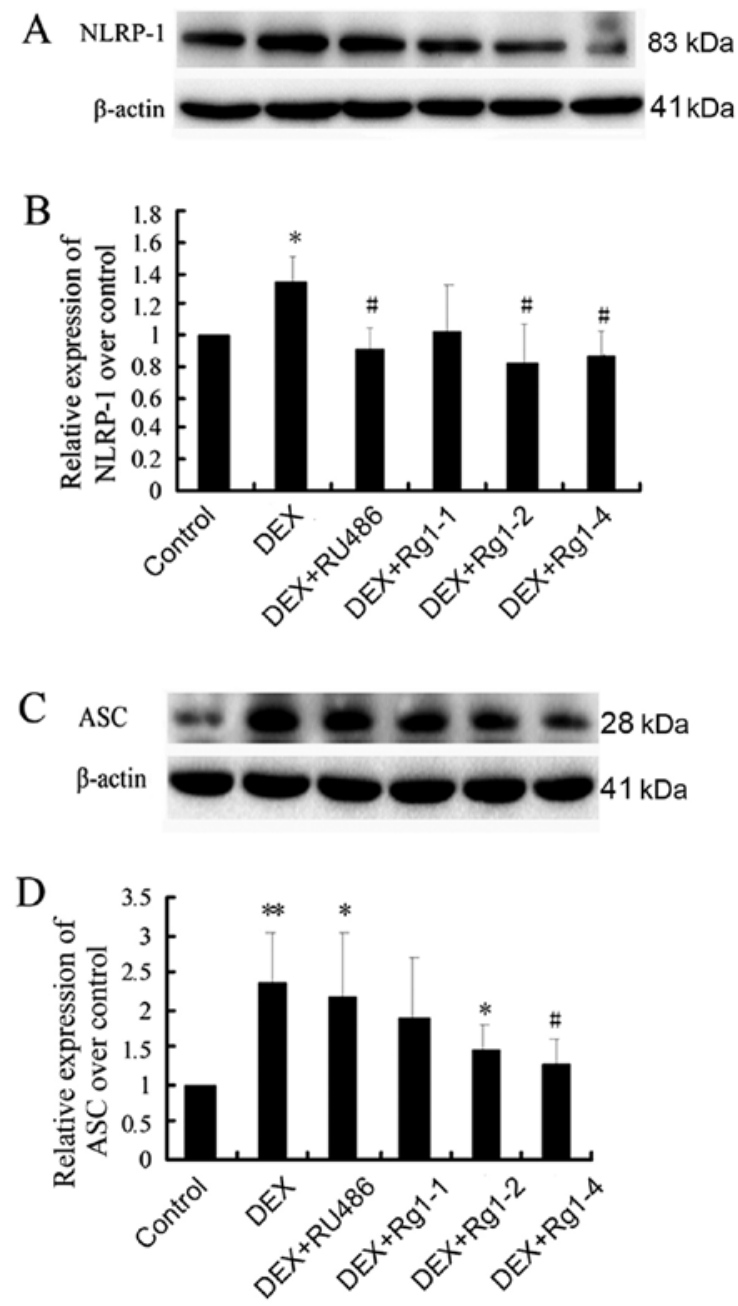

Figure 6. Effects of ginsenoside Rg1 (Rg1) on NLRP-1 and ASC expression in the frontal cortex and hippocampus in chronic DEX-exposed mice (immunoblotting). (A) The immunoblot result of NLRP-1 and (B) quantitative analysis of NLRP-1 expression over the control. (C) The immunoblot result of ASC and (D) quantitative analysis of ASC expression over the control. Results are expressed as mean $\pm \mathrm{SD}$, NLRP-1, $\mathrm{n}=3$; ASC, $\mathrm{n}=4$. ${ }^{*} \mathrm{P}<0.05$, compared with the control group; ${ }^{\text {}} \mathrm{P}<0.05$, compared with the DEX group. DEX, dexamethasone.

expression and decreased IL-1 $\beta$ and IL-18 expression in brain tissue. The results suggest that Rg1 may inhibit chronic DEX exposure-induced neuronal inflammation, which may prevent the generation and progression of $\mathrm{AD}$.

Growing evidence has linked high levels of GCs with neurodegenerative diseases, such as vulnerability to depression, AD and PD $(28,29)$. Numerous studies have shown that chronic high concentrations of GCs have adverse effects on neurons, and even cause hippocampal neuronal death $(3,5,6)$. DEX is a type of synthetic glucocorticoid. It has been reported that chronic exposure to DEX $(5 \mathrm{mg} / \mathrm{kg})$ can induce learning and memory impairment and mimic GC-induced neurodegenerative diseases $(16,17,30)$. In our previous studies, we found that both chronic DEX $(5 \mathrm{mg} / \mathrm{kg})$ exposure and restrain stress induced cognition impairments and neuronal damage in the hippocampus and cortex in mice $(19,31)$. Our most recent study revealed that DEX $(5 \mathrm{mg} / \mathrm{kg})$ exposure for 28 days significantly increased expression of NLRP-1 and accelerated neurodegeneration in the hippocampus and frontal cortex in 
A

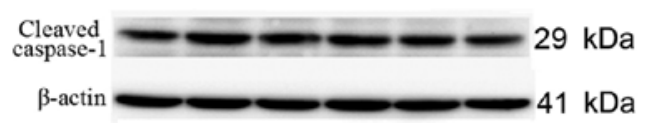

$\mathrm{B}$

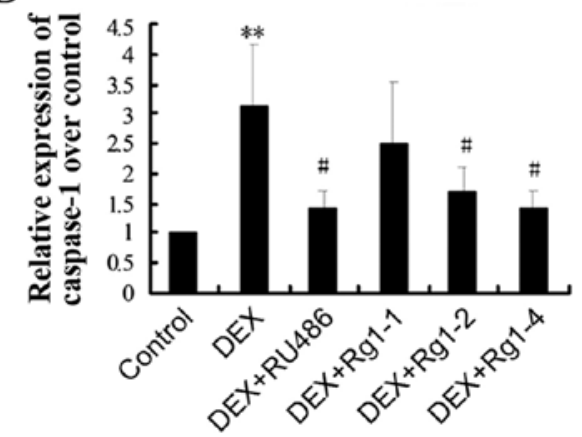

$\mathrm{C}$

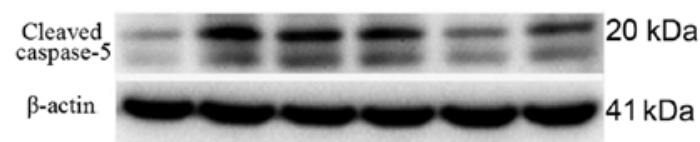

$\mathrm{D}$

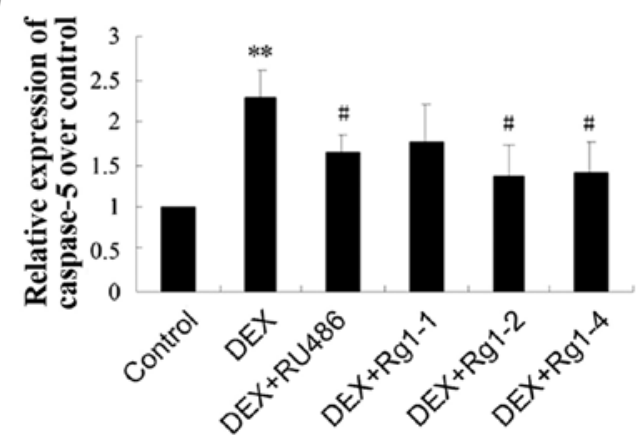

Figure 7. Effects of ginsenoside Rg1 (Rg1) on cleaved caspase-1 and caspase-5 expression in the frontal cortex and hippocampus in chronic DEX-exposed mice (immunoblotting). (A) The immunoblot result of caspase-1 and (B) quantitative analysis of caspase-1 expression over the control. (C) The immunoblot result of caspase -5 and (D) quantitative analysis of caspase-5 expression over the control. Results are expressed as mean $\pm \mathrm{SD}$, caspase $-1, \mathrm{n}=4$; caspase -5 , $\mathrm{n}=3$. $\mathrm{P}<0.05$, compared with the control group; ${ }^{\#} \mathrm{P}<0.05$ compared with the DEX exposure group. DEX, dexamethasone.

A

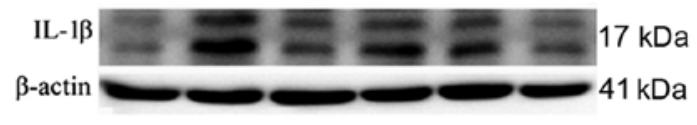

$\mathrm{B}$

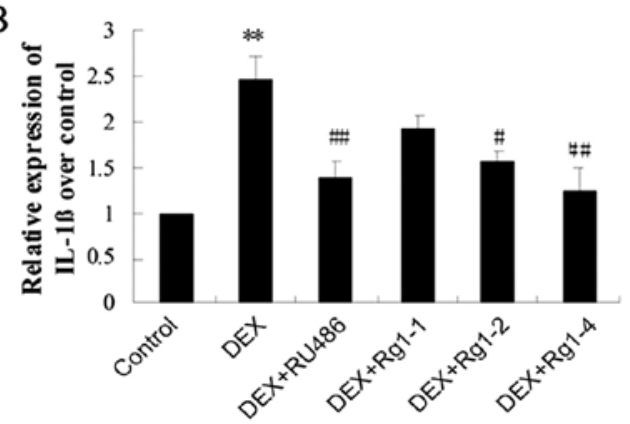

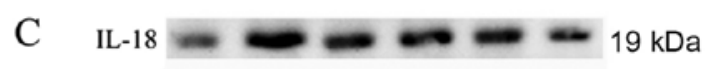

$\beta$-actin $41 \mathrm{kDa}$

$\mathrm{D}$

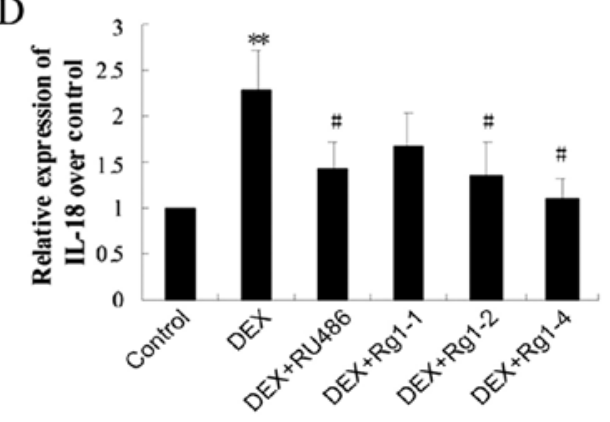

Figure 8. Effects of ginsenoside Rg1 (Rg1) on mature IL-1 $\beta$ and IL-18 expression in the frontal cortex and hippocampus in chronic DEX-exposed mice (immunoblotting). (A) The immunoblot result of IL-1 $\beta$ and (B) quantitative analysis of IL-1 $\beta$ expression over the control. (C) The immunoblot result of IL-18 and (D) quantitative analysis of IL-18 expression over the control. Results are expressed as mean $\pm \mathrm{SD}, \mathrm{n}=3$. ${ }^{* *} \mathrm{P}<0.01$ compared with the control group. ${ }^{\#} \mathrm{P}<0.05$, ${ }^{\# \#} \mathrm{P}<0.01$ compared with the DEX group. DEX, dexamethasone.

mice (18). However, currently, there are no effective drugs for preventing chronic GC exposure-mediated neuronal damage. It was found that ginsenoside $\mathrm{Rg} 1$ attenuated the inflammation, neuronal apoptosis and alleviated the cognitive impairment in an AD model $(32,33)$. Our previous study also showed that Rg1 could protect against learning and memory impairments and ROS oxidative damage in chronic restrain stress-exposed mice (19). Yet, it is still unclear whether Rg1 could alleviate chronic GC-induced cognitive impairment and neuronal inflammation damage.

Behavioral tests play an important role in evaluating the ability of recognition in animal models of cognitive dysfunction (34). The open field test is generally used to evaluate locomotor activity and spontaneous exploration in a novel environment (35). In the present study, we observed that chronic DEX-exposed animals showed impaired motor and exploration activities such as reduced number of lines crossed and stand up events in the open field test. Rg1 ( 2 and $4 \mathrm{mg} / \mathrm{kg}$ ) significantly increased the number of lines crossed and stand up movements of the mice. The NOR test is also widely used to investigate memory alterations. As Ennaceur (36) reported, animals were placed to an arena with a familiar and a novel object. He found that the animals approached frequently and spent more time exploring the novel object than the familiar one. In the present study, we found that DEX exposure for 28 days significantly decreased the entering number and the exploring time in the novel object zone. Compared with the DEX treatment group, RU486 and Rg1 (2 and $4 \mathrm{mg} / \mathrm{kg}$ ) treatment increased the entering number and the exploring time in the novel object zone. These results suggest that Rg1 may alleviate the cognitive dysfunction induced by chronic GC exposure.

As a cytoskeletal protein, MAP2 is a marker of structural integrity in neurons (37). It has been observed that there is 
a significant reduction in MAP2 in the cortex and hippocampus in elder rats (38). The present study showed that DEX $(5 \mathrm{mg} / \mathrm{kg}$ ) exposure for 28 days significantly induced neuronal damage; the neurons showed significant acidophilia degeneration and nuclear condensation in the cortex and hippocampus CA1, CA3, and the expression of MAP2 was also significantly reduced in the frontal cortex and hippocampus. Treatment with Rg1 (2 and $4 \mathrm{mg} / \mathrm{kg}$ ) and RU486 significantly alleviated the histological changes, increased the expression of MAP2, and attenuated the neuronal injury in the frontal cortex and hippocampus CA1 and CA3.

Neuroinflammation has emerged as an important cause of the cognitive decline during aging and $\operatorname{AD}(25,26,39)$. GCs are commonly used as anti-inflammatory and immunosuppressive agents in the clinic. However, research has showed that GCs also have a proinflammatory action $(40,41)$. The GR, as a member of the nuclear receptor superfamily, plays a key role in regulated the actions of GCs (42). GR contributes to the regulation of proinflammatory molecules at the level of gene transcription (43). Sun et al (13) reported that LPS treatment slightly downregulated GR expression. Rg1 treatment significantly increased the GR expression compared to the LPS group. The GR antagonist RU486 inhibited the neuroprotective effects of $\mathrm{Rg} 1$, indicating that the anti-inflammatory effects of $\mathrm{Rg} 1$ are dependent on GR. Du et al (44) reported that Rg1, a novel GR agonist of plant origin, possesses GC and estrogen-like activities and can effectively inhibit acute and chronic inflammation, but it does not cause an adverse reaction as noted with DEX. In the present study, we found that DEX ( $5 \mathrm{mg} / \mathrm{kg})$ exposure for 28 days significantly downregulated GR expression and increased IL-1 $\beta$ and IL-18 expression in the hippocampus and frontal cortex in mice. Rg1 and RU486 increased GR expression and reduced IL-1 $\beta$ and IL-18 expression in the hippocampus and frontal cortex in mice. The results suggest that both Rg1 and RU486 can increase GR expression and are involved in anti-inflammatory effects in chronic DEX-exposed mice. However, the mechanisms through which Rg1 inhibits the downregulation of GR expression induced by DEX are still unclear and warrant further study.

The inflammasome-associated pathway plays an important role in the pathogenesis of neurodegenerative diseases. The NLRP1 inflammasome is a multi-protein complex which consists of NLRP1, the adaptor ASC and caspase-1 (45). NLRP1 inflammasomes mediate activation of caspase-1 which promotes cleavage of mature proinflammatory cytokines from pro-IL-1 $\beta$ and pro-IL-18 into IL-1 $\beta$ and IL-18 (46). The adaptor ASC is a critical component of inflammasomes by linking NLRPs to caspase-1 activation (47). Therefore, activation of inflammasomes provides a molecular platform for caspase- 1 activation which promotes IL-1 $\beta$ and IL-18 release $(48,49)$. Caspase- 5 is also a proinflammatory cysteine protease. Caspase- 5 together with caspase- 1 are components of the NLRP1 inflammasome complex and enhance activation of caspase-1 (50). The present study showed that DEX exposure for 28 days significantly increased the expression levels of NLRP-1, caspase-1, caspase-5, ASC, IL-1 $\beta$ and IL-18 in the hippocampus and frontal cortex brain tissue. Rg1 and RU486 significantly decreased the expression levels of NLRP-1, caspase-1, caspase-5, ASC, IL-1 $\beta$ and IL-18 in the hippocampus and frontal cortex brain tissue. These data suggest that Rg1 may suppress neuroinflammation and inhibit chronic DEX exposure-induced inflammation injury in the hippocampus and frontal cortex.

In summary, the present study suggests that chronic GC exposure induces neurodegeneration and NLRP-1 inflammasome activation in the hippocampus and frontal cortex. Rg1 protects against the neuroinflammation and neuronal damage induced by chronic DEX exposure. Additionally, the inhibition of NLRP-1 inflammasomes was involved in the action mechanisms of $\operatorname{Rg} 1$ in this experimental model. However, this study only offered an experimental basis for Rg1 in the treatment of chronic DEX exposure, and other related molecular mechanisms of Rg1 in regards to DEX exposure warrant further investigation.

\section{Acknowledgements}

The present study was financially supported by grants from the National Nature Science Foundation of China (81371329 and 81671384) and the Natural Science Foundation of Anhui Province Education Department (KJ2015A298, KJ2016A357). We thank Bao Li and Li Gui (Synthetic Laboratory of Basic Medicine College, Anhui Medical University) for their excellent technical assistance.

\section{References}

1. Joshi YB, Chu J and Praticò D: Stress hormone leads to memory deficits and altered tau phosphorylation in a model of Alzh-eimer's disease. J Alzheimers Dis 31: 167-176, 2012.

2. Moceri VM, Kukull WA, Emanuel I, van Belle G and Larson EB Early-life risk factors and the development of Alzheimer's disease. Neurology 54: 415-420, 2000.

3. Csernansky JG, Dong H, Fagan AM, Wang L, Xiong C, Holtzman DM and Morris JC: Plasma cortisol and progression of dementia in subjects with Alzheimer-type dementia. Am J Psychiatry 163: 2164-2169, 2006.

4. Aznar S and Knudsen GM: Depression and Alzheimer's disease: Is stress the initiating factor in a common neuropathological cascade? J Alzheimers Dis 23: 177-193, 2011.

5. Kleen JK, Sitomer MT, Killeen PR and Conrad CD: Chronic stress impairs spatial memory and motivation for reward without disrupting motor ability and motivation to explore. Behav Neurosci 120: 842-851, 2006.

6. Conrad CD, McLaughlin KJ, Harman JS, Foltz C, Wieczorek L, Lightner E and Wright RL: Chronic glucocorticoids increase hippocampal vulnerability to neurotoxicity under conditions that produce CA3 dendritic retraction but fail to impair spatial recognition memory. J Neurosci 27: 8278-8285, 2007.

7. MacPherson A, Dinkel K and Sapolsky R: Glucocorticoids worsen excitotoxin-induced expression of pro-inflammatory cytokines in hippocampal cultures. Exp Neurol 194: 376-383, 2005.

8. Kim EJ, Pellman B and Kim JJ: Stress effects on the hippocampus: A critical review. Learn Mem 22: 411-416, 2015.

9. Rausch WD, Liu S, Gille G and Radad K: Neuroprotective effects of ginsenosides. Acta Neurobiol Exp (Wars) 66: 369-375, 2006.

10. Xie CL, Wang WW, Xue XD, Zhang SF, Gan J and Liu ZG: A systematic review and meta-analysis of Ginsenoside-Rg1 (G-Rg1) in experimental ischemic stroke. Sci Rep 5: 7790, 2015.

11. Zhang X, Wang J, Xing Y, Gong L, Li H, Wu Z, Li Y, Wang J, Wang Y, Dong L, et al: Effects of ginsenoside Rg1 or $17 \beta$-estradiol on a cognitively impaired, ovariectomized rat model of Alzheimer's disease. Neuroscience 220: 191-200, 2012.

12. Baulieu EE: Contragestion and other clinical applications of RU 486, an antiprogesterone at the receptor. Science 245: 1351-1357, 1989.

13. Sun XC, Ren XF, Chen L, Gao XQ, Xie JX and Chen WF: Glucocorticoid receptor is involved in the neuroprotective effect of ginsenoside $\operatorname{Rg} 1$ against inflammation-induced dopaminergic neuronal degeneration in substantia nigra. J Steroid Biochem Mol Biol 155 (Pt A): 94-103, 2016. 
14. Behl C, Lezoualc'h F, Trapp T, Widmann M, Skutella T and Holsboer F: Glucocorticoids enhance oxidative stress-induced cell death in hippocampal neurons in vitro. Endocrinology 138: 101-106, 1997.

15. McCullers DL, Sullivan PG, Scheff SW and Herman JP. Mifepristone protects CA1 hippocampal neurons following traumatic brain injury in rat. Neuroscience 109: 219-230, 2002.

16. Green KN, Billings LM, Roozendaal B, McGaugh JL and LaFerla FM: Glucocorticoids increase amyloid-beta and tau pathology in a mouse model of Alzheimer's disease. $\mathrm{J}$ Neurosci 26: 9047-9056, 2006.

17. Maloney SE, Noguchi KK, Wozniak DF, Fowler SC and Farber NB: Long-term effects of multiple glucocorticoid exposures in neonatal mice. Behav Sci (Basel) 1: 4-30, 2011.

18. Hu W, Zhang Y, Wu W, Yin Y, Huang D, Wang Y, Li W and Li W: Chronic glucocorticoids exposure enhances neurodegeneration in the frontal cortex and hippocampus via NLRP-1 inflammasome activation in male mice. Brain Behav Immun 52: 58-70, 2016.

19. Wang Y, Kan H, Yin Y, Wu W, Hu W, Wang M, Li W and $\mathrm{Li} \mathrm{W}$ : Protective effects of ginsenoside $\mathrm{Rg} 1$ on chronic restraint stress induced learning and memory impairments in male mice. Pharmacol Biochem Behav 120: 73-81, 2014

20. Koros E, Piasecki J, Kostowski W and Bienkowski P: Saccharin drinking rather than open field behaviour predicts initial ethanol acceptance in Wistar rats. Alcohol Alcohol 33: 131-140, 1998.

21. de Senna PN, Ilha J, Baptista PP, do Nascimento PS, Leite MC, Paim MF, Gonçalves CA, Achaval M and Xavier LL: Effects of physical exercise on spatial memory and astroglial alterations in the hippocampus of diabetic rats. Metab Brain Dis 26: 269-279, 2011.

22. Frye CA, Paris JJ and Rhodes ME: Engaging in paced mating, but neither exploratory, anti-anxiety, nor social behavior, increases 5alpha-reduced progestin concentrations in midbrain, hippocampus, striatum, and cortex. Reproduction 133: 663-674, 2007.

23. Antunes M and Biala G: The novel object recognition memory: Neurobiology, test procedure, and its modifications. Cogn Process 13: 93-110, 2012

24. Zotova E, Bharamb---e V, Cheaveau M, Morgan W, Holmes C, Harris S, Neal JW, Love S, Nicoll JA and Boche D: Inflammatory components in human Alzheimer--'s disease and after active amyloid- $\beta 42$ immunization. Brain 136: 2677-2696, 2013.

25. Calsolaro V and Edison P: Neuroinflammation in Alzheimer's disease: Current evidence and future directions. Alzheimers Dement 12: 719-732, 2016.

26. Morales I, Guzmán-Martínez L, Cerda-Troncoso C, Farías GA and Maccioni RB: Neuroinflammation in the pathogenesis of Alzheimer's disease. A rational framework for the search of novel therapeutic approaches. Front Cell Neurosci 8: 112, 2014.

27. Jha S, Srivastava SY, Brickey WJ, Iocca H, Toews A, Morrison JP, Chen VS, Gris D, Matsushima GK and Ting JP: The inflammasome sensor, NLRP3, regulates CNS inflammation and demyelination via caspase-1 and interleukin-18. J Neurosci 30: 15811-15820, 2010.

28. Sotiropoulos I, Catania C, Pinto LG, Silva R, Pollerberg GE, Takashima A, Sousa N and Almeida OF: Stress acts cumulatively to precipitate Alzheimer's disease-like tau pathology and cognitive deficits. J Neurosci 31: 7840-7847, 2011.

29. Chen KC, Blalock EM, Curran-Rauhut MA, Kadish I, Blalock SJ, Brewer L, Porter NM and Landfield PW: Glucocorticoid-dependent hippocampal transcriptome in male rats: Pathway-specific alterations with aging. Endocrinology 154 $2807-2820,2013$

30. Danilczuk Z, Ossowska G, Lupina T, Cieślik K and Zebrowska-Łupina I: Effect of NMDA receptor antagonists on behavioral impairment induced by chronic treatment with dexamethasone. Pharmacol Rep 57: 47-54, 2005.

31. Li WZ, Li WP, Yao YY, Zhang W, Yin YY, Wu GC and Gong HL: Glucocorticoids increase impairments in learning and memory due to elevated amyloid precursor protein expression and neuronal apoptosis in 12-month old mice. Eur J Pharmacol 628: $108-115,2010$.
32. Kril JJ, Patel S, Harding AJ and Halliday GM: Neuron loss from the hippocampus of Alzheimer's disease exceeds extracellular neurofibrillary tangle formation. Acta Neuropathol 103: 370-376, 2002.

33. Yang Y, Li X, Zhang L, Liu L, Jing G and Cai H: Ginsenoside Rg1 suppressed inflammation and neuron apoptosis by activating PPAR $\gamma / \mathrm{HO}-1$ in hippocampus in rat model of cerebral ischemia-reperfusion injury. Int J Clin Exp Pathol 8: 2484-2494, 2015.

34. Baxter MG: 'I've seen it all before': Explaining age-related impairments in object recognition. Theoretical comment on Burke et al (2010). Behav Neurosci 124: 706-709, 2010.

35. Wi S, Yu JH, Kim M and Cho SR: In vivo expression of reprogramming factors increases hippocampal neurogenesis and synaptic plasticity in chronic hypoxic-ischemic brain injury. Neural Plast 2016: 2580837, 2016 (Epub ahead of print).

36. Ennaceur A: One-trial object recognition in rats and mice: Methodological and theoretical issues. Behav Brain Res 215: 244-254, 2010.

37. Di Stefano G, Casoli T, Fattoretti P, Gracciotti N, Solazzi M and Bertoni-Freddari C: Distribution of map2 in hippocampus and cerebellum of young and old rats by quantitative immunohistochemistry. J Histochem Cytochem 49: 1065-1066, 2001.

38. Chauhan N and Siegel G: Age-dependent organotypic expression of microtubule-associated proteins (MAP1, MAP2, and MAP5) in rat brain. Neurochem Res 22: 713-719, 1997.

39. Sartori AC, Vance DE, Slater LZ and Crowe M: The impact of inflammation on cognitive function in older adults: Implications for healthcare practice and research. J Neurosci Nurs 44: 206-217, 2012.

40. Frank MG, Miguel ZD, Watkins LR and Maier SF: Prior exposure to glucocorticoids sensitizes the neuroinflammatory and peripheral inflammatory responses to E. coli lipopolysaccharide. Brain Behav Immun 24: 19-30, 2010.

41. Hermoso MA, Matsuguchi T, Smoak K and Cidlowski JA: Glucocorticoids and tumor necrosis factor alpha cooperatively regulate toll-like receptor 2 gene expression. Mol Cell Biol 24: 4743-4756, 2004.

42. Carter BS, Meng F and Thompson RC: Glucocorticoid treatment of astrocytes results in temporally dynamic transcriptome regulation and astrocyte-enriched mRNA changes in vitro. Physiol Genomics 44: 1188-1200, 2012.

43. Chrousos GP: Stress and disorders of the stress system. Nat Rev Endocrinol 5: 374-381, 2009.

44. Du J, Cheng B, Zhu X and Ling C: Ginsenoside Rg1, a novel glucocorticoid receptor agonist of plant origin, maintains glucocorticoid efficacy with reduced side effects. J Immunol 187: 942-950, 2011

45. Martinon F and Tschopp J: NLRs join TLRs as innate sensors of pathogens. Trends Immunol 26: 447-454, 2005.

46. Franchi L, Eigenbrod T, Muñoz-Planillo R and Nuñez G: The inflammasome: A caspase-1-activation platform that regulates immune responses and disease pathogenesis. Nat Immunol 10: 241-247, 2009.

47. Mariathasan S, Newton K, Monack DM, Vucic D, French DM, Lee WP, Roose-Girma M, Erickson S and Dixit VM: Differential activation of the inflammasome by caspase-1 adaptors ASC and Ipaf. Nature 430: 213-218, 2004.

48. Stutz A, Golenbock DT and Latz E: Inflammasomes: Too big to miss. J Clin Invest 119: 3502-3511, 2009.

49. Fernandes-Alnemri T, Wu J, Yu JW, Datta P, Miller B, Jankowski W, Rosenberg S, Zhang J and Alnemri ES: The pyroptosome: A supramolecular assembly of ASC dimers mediating inflammatory cell death via caspase-1 activation. Cell Death Differ 14: 1590-1604, 2007.

50. Salminen A, Ojala J, Suuronen T, Kaarniranta K and Kauppinen A: Amyloid-beta oligomers set fire to inflammasomes and induce Alzheimer's pathology. J Cell Mol Med 12 (6A): 2255-2262, 2008. 